\title{
A contraction theory-based analysis of the stability of the Extended Kalman Filter
}

\author{
Silvère Bonnabel* Jean-Jacques Slotine ${ }^{\dagger}$
}

October 30, 2018

\begin{abstract}
The contraction properties of the Extended Kalman Filter, viewed as a deterministic observer for nonlinear systems, are analyzed. This yields new conditions under which exponential convergence of the state error can be guaranteed. As contraction analysis studies the evolution of an infinitesimal discrepancy between neighboring trajectories, and thus stems from a differential framework, the sufficient convergence conditions are different from the ones that previously appeared in the literature, which were derived in a Lyapunov framework. This article sheds another light on the theoretical properties of this popular observer.
\end{abstract}

Keywords Nonlinear asymptotic observer, Extended Kalman Filter, Contraction theory.

\section{Introduction}

Since the seminal work of Kalman and Bucy [9] and Luenberger [14], the problem of building observers for deterministic linear systems has been laid on firm theoretical ground. Yet, when the system is nonlinear, there is no general methods to tackle observer design. Over the last decades, nonlinear observer design has been an active field of research, and several methods have emerged for attacking some specific nonlinearities. In the engineering world, the most popular method is the so-called Extended Kalman Filter (EKF), a natural extension of the Kalman filter. The principle is to linearize the system around the trusted (i.e. estimated) trajectory of the system, build a Kalman filter for this time-varying linear model, and implement it on the nonlinear model. The EKF is known to yield good results in practice when the guess on the initial state is close enough to the actual state, but possesses no guarantee of convergence in the general case, and indeed can diverge.

Since the 1990's, several papers have addressed the convergence properties of the EKF viewed as a deterministic observer. Several conditions under which the estimation error converges to zero have been derived in, e.g., 1, 16, 2, 15. In each case, a first set of conditions on the observability and controllability of the system ensures the boundedness of the solution of the Riccati equation and of its inverse, and a second set of conditions ensure in this case the convergence of the estimation error to zero. Roughly speaking, the latter conditions require either the initial estimation error to be small, proving the EKF is a local observer, or the system to be very weakly nonlinear.

\footnotetext{
${ }^{*}$ Robotics Center, Unité Mathématiques et Systèmes, Ecole des Mines ParisTech, 75272 Paris, France (silvere.bonnabel@mines-paristech.fr).

${ }^{\dagger}$ Department of Mechanical Engineering, Massachusetts Institute of Technology, MA, 02139 USA (jjs@mit.edu).
} 
In this paper, the convergence properties of the EKF are studied using contraction theory 12 and in particular the notion of virtual systems [17 and virtual observers [8. Historically, ideas closely related to contraction can be traced back to [11, 7, 4, (see e.g. [13 for a more exhaustive list of references). In the present case the idea is as follows: instead of studying directly the evolution of the discrepency, in the sense of a Lyapunov function, between the estimated state and the true state, contraction theory allows to study the evolution of the discrepancy between two nearby trajectories of the EKF, in the sense of a given metric. It is shown that, in a finite region and under some conditions, two nearby trajectories tend exponentially towards each other. As a result, the EKF is a dynamical system which exponentially forgets its initial condition, a very desirable property for a filter. The fact that the estimation error tends exponentially to the true state appears then as a mere consequence of the contraction properties of the filter. Even though the Lyapunov approach and the contraction approach are based on very similar metrics, the convergence results obtained in this paper differ from those of the literature.

The main contributions of this paper are threefold. First, the paper studies the stability properties of the EKF from the perspective of contraction theory. This offers an alternative viewpoint to the usual Lyapunov approach, extending the preliminary results on linear timevarying systems of [8]. In turn, this perspective allows simple new convergence results to be derived in this context (see in particular Theorem 1 and its corollary). Finally, some of the results are closely related to existing recent literature, showing both their similarities and their potential strengths.

The paper is organized as follows. In Section 2, some new general contraction results are derived. Section 3 builds upon those results to derive bounds on the size of the contraction region and the convergence rate of the EKF. Finally, Section 4 discusses some links with previous work on the stability of the EKF.

\section{General contraction results}

Consider the following nonlinear deterministic system

$$
\begin{aligned}
\frac{d}{d t} x & =f(x, t) \\
y_{m} & =h(x, t)
\end{aligned}
$$

where $x \in \mathbb{R}^{n}$ is the state, $y_{m} \in \mathbb{R}^{p}$ is the measured output, and $f, h$ are smooth. The EKF equations are given by

$$
\begin{aligned}
& \frac{d}{d t} \hat{x}=f(\hat{x}, t)-P C(\hat{x}, t)^{T} R^{-1}\left(h(\hat{x}, t)-y_{m}(t)\right) \\
& \frac{d}{d t} P=A(\hat{x}, t) P(t)+P(t) A(\hat{x}, t)^{T}+Q-P(t) C(\hat{x}, t)^{T} R^{-1} C(\hat{x}, t) P(t)
\end{aligned}
$$

where $A(x, t)=\frac{\partial f}{\partial x}(x, t)$, and $C(x, t)=\frac{\partial h}{\partial x}(x, t)$. In the stochastic theory of Kalman linear filtering, $Q$ and $R$ represent the covariances of the respectively drift noise and measurement noise. In a deterministic and nonlinear setting as the one considered in the present paper, they can be viewed more prosaically as design parameters where $Q^{-1}$ represents the confidence in the trusted model (11) and $R^{-1}$ the confidence in the measurements (2). The present analysis relies on the following assumption.

Assumption 1 From now on we will systematically assume there exist $\underline{p}, \bar{p}>0$ such that $p I \leq P(t) \leq \bar{p} I$. Moreover, for simplicity we assume that $Q$ is fixed and invertible, and we

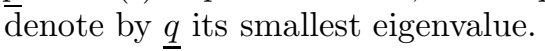


The latter assumption on $P(t)$ appears in most papers dealing with the stability of the EKF, e.g. 1, 5, 16. It is well known that this assumption is verified as soon as the system $\dot{\xi}=A(\hat{x}(t), t) \xi, \eta=C(\hat{x}(t), t) \xi$ is uniformly detectable. This is of course a very strong prerequisite on the behavior of the filter. Yet, note that this assumption can advantageously be checked by the user without any knowledge of the true trajectory. To the authors' best knowledge, very few papers have addressed the stability of the EKF without referring to Assumption 1: see [10] (and more generally high gain observers techniques [6]) where local convergence results are derived under some different, yet rather restrictive, assumptions.

Let $K(t)=P C(\hat{x}, t)^{T} R^{-1}$ denote the Kalman gain. Consider the "virtual" system [8]

$$
\frac{d}{d t} z=f(z, t)-K(t)\left(h(z, t)-y_{m}(t)\right)
$$

The solution $x(t)$ of the true system (1) is a particular solution of the virtual system, since for all $t \geq 0$ we have $h(x(t), t)-y_{m}(t)=0$. The solution to the EKF equations (3)-(4) is obviously another particular solution of the virtual system. As a result, if it can be proven the distance between two arbitrary trajectories of this system tends to zero, the convergence of the estimation error $\hat{x}-x$ to zero will follow. In turn, this can be achieved by seeking conditions under which the virtual system (5) is contracting.

Let us define a metric for the virtual system (5) by choosing, similarly to the linear time-varying case considered in $[8$, the squared length

$$
\|\delta z\|_{P^{-1}}^{2}=\delta z^{T} P^{-1} \delta z
$$

where $P(t)$ is a solution of the Riccati equation (4) associated to the trajectory of the estimated state $\hat{x}(t)$. We have

$$
\begin{aligned}
\frac{d}{d t}\left(\delta z^{T} P^{-1} \delta z\right)= & \left(\frac{d}{d t} \delta z\right)^{T} P^{-1} \delta z+\delta z \dot{P}^{-1} \delta z+\delta z P^{-1}\left(\frac{d}{d t} \delta z\right) \\
=\delta & \delta z^{T}\left[(A(z, t)-K(t) C(z, t))^{T} P^{-1}+\dot{P}^{-1}\right. \\
& \left.+P^{-1}(A(z, t)-K(t) C(z, t))\right] \delta z
\end{aligned}
$$

Using the fact that $\dot{P}^{-1}=-P^{-1} \dot{P} P^{-1}$ where $\dot{P}$ is given by (4), and that

$$
\begin{aligned}
& {[C(z, t)-C(\hat{x}, t)] R^{-1}[C(z, t)-C(\hat{x}, t)]} \\
& \quad=C^{T}(z, t) R^{-1} C(z, t)-C^{T}(\hat{x}, t) R^{-1} C(z, t)-C^{T}(z, t) R^{-1} C(\hat{x}, t)+C^{T}(\hat{x}, t) R^{-1} C(\hat{x}, t)
\end{aligned}
$$

we finally have

$$
\frac{d}{d t}\left(\delta z^{T} P^{-1} \delta z\right)=\delta z^{T} P^{-1} M P^{-1} \delta z
$$

where $M=P \tilde{A}^{T}+\tilde{A} P+P \tilde{C}^{T} R^{-1} \tilde{C} P-P C^{T} R^{-1} C P-Q$, where we let $\tilde{A}(z, t)=A(z, t)-$ $A(\hat{x}, t)$ and $\tilde{C}(z, t)=C(z, t)-C(\hat{x}, t)$, and where $C$ denotes the matrix $C(z, t)$.

\section{$2.1 \quad$ Main result}

Given two symmetric matrices $P_{1}, P_{2}$ we define a partial order letting $P_{1} \leq P_{2}$ if $P_{2}-P_{1}$ is positive semidefinite. We have the following preliminary result:

Lemma 1 Let $0 \leq \gamma<q /(2 \bar{p})$. For each time $t \geq 0$ there exists $r(t)>0$ such that for all $z$ satisfying $\|z-\hat{x}(t)\| \leq r \overline{(t})$ we have

$$
P \tilde{A}^{T}+\tilde{A} P+P \tilde{C}^{T} R^{-1} \tilde{C} P \leq Q-2 \gamma P+P C^{T} R^{-1} C P
$$


Proof The inequality is obviously verified for $z=\hat{x}$ as the right member of (9) is a positive definite matrix. As $f, h$ are smooth, the inequality holds in a neighborhood of $\hat{x}$.

At any time, the vectors $z$ lying within a distance at most $r(t)$ of $\hat{x}(t)$ are contained in the contraction region, as for those vectors equality (8) becomes the contraction inequality

$$
\frac{d}{d t}\left(\delta z^{T} P^{-1} \delta z\right) \leq-2 \gamma\left(\delta z^{T} P^{-1} \delta z\right)
$$

This means the squared distance in the sense of metric (6) between two neighboring trajectories in this ball will tend to reduce, with a rate of change $\gamma$. This leads to the following general result:

Theorem 1 Assume there exists $0<\rho=\inf \{r(t), t \geq 0\}$. Any trajectory of the system (51) that starts in the ball of center $\hat{x}(0)$ and constant radius $\rho / \sqrt{\bar{p}}$ with respect to the metric (6) remains in a ball of radius $\rho / \sqrt{\bar{p}}$ centered at the trajectory $\hat{x}(t)$ of the Extended Kalman Filter (3)-(41), and converges exponentially to this trajectory in the sense of the metric (6) with a time constant $1 / \gamma$ for the exponential decay.

Mathematically, the theorem's result can be expressed as follows. Let $d_{P^{-1}}$ be the geodesic distance associated with the metric (6). Let $z_{1}(t), z_{2}(t)$ be the flows associated to the system (5) with initial conditions satisfying $d_{P^{-1}}\left(z_{i}(0), \hat{x}(0)\right) \leq \rho / \sqrt{\bar{p}}$ for $i=1,2$. Then for all times $t \geq 0$

$$
d_{P^{-1}}\left(z_{1}(t), z_{2}(t)\right) \leq e^{-\frac{t}{\gamma}} d_{P^{-1}}\left(z_{1}(0), z_{2}(0)\right)
$$

Proof The theorem is a straightforward application of the Theorem 2 of [12] which states that any trajectory which starts in a ball of constant radius with respect to the metric centered at a given trajectory and at all times in the contraction region with respect to the metric, remains in that ball and converges exponentially to this trajectory, which is a natural result in the theory of contracting flows (see e.g. [11]). Indeed, $(z-\hat{x})^{T} P^{-1}(z-\hat{x}) \leq \rho^{2} / \bar{p}$ implies $\|z-\hat{x}\| \leq \rho$ so $z$ is contained in the contraction region by Lemma 1 .

Remark 1 Note that, if the system is linear, we have $\tilde{A}(z, t) \equiv \tilde{C}(z, t) \equiv 0$ for all $z, t$, and we recover the fact that the deterministic Kalman filter for linear systems globally exponentially converges under Assumption 1.

\subsection{Particular case of a linear output map}

The theorem implies an interesting result in the common case of linear output maps. In many nonlinear systems of engineering interest, the system output consists of an incomplete measurement of the state vector. For instance, the output can be temperature or concentrations in chemical reactors, currents in induction machines, position or velocity in mechanical systems. Formally, this means that the output map is linear, i.e. $h(x)=C x$, implying $\tilde{C}(z, t) \equiv 0$ for all $z, t$. Let then $\lambda_{\max }(\cdot)$ denote the largest eigenvalue of a symmetric matrix, and let $\gamma \geq 0$. The following result is an immediate consequence of Theorem 1 .

Corollary 1 Assume that the output map is linear, and that $\lambda_{\max }\left(\tilde{A}(z, t) P(t)+P(t) \tilde{A}(z, t)^{T}\right) \leq$ $\underline{q}-2 \gamma \bar{p}$ for all $z, t$. Then the Extended Kalman Filter (3)-(4) is globally exponentially convergent with a time constant $1 / \gamma$ for the exponential decay.

This result shows that contraction analysis can yield new types of conditions under which exponential convergence of the EKF is guaranteed. Indeed, under the assumptions of Corollary 1, the EKF will converge globally without the standard requirement that the Hessian of the coordinates of $f$ is uniformly bounded. However, in a more general context, this standard requirement will still be needed as illustrated in the next section. 


\section{A sufficient condition for exponential convergence}

We now derive a lower bound on the size of the contraction region of Theorem 1 . This result relies on usual assumptions on boundedness of second derivatives $\frac{\partial^{2} f}{\partial x^{2}}, \frac{\partial^{2} h}{\partial x^{2}}$ around the observer trajectory $\hat{x}(t), t \geq 0$. We let $\|\cdot\|$ and $\|\cdot\|$ denote the norms on resp. matrices and tensors induced by the Euclidean norm on vectors.

Assumption 2 There are positive numbers $\alpha, \kappa_{A}, \kappa_{C}$ such that for all $z$ satisfying $\|\hat{x}-z\| \leq$ $\alpha$ and all $t \geq 0$ we have $\left\|\frac{\partial^{2} f}{\partial x^{2}}(z, t)\right\| \leq \kappa_{A}$ and $\left\|\frac{\partial^{2} h}{\partial x^{2}}(z, t)\right\| \leq \kappa_{C}$.

Under Assumptions 1 and 2, one can derive the following local exponential stability result:

Corollary 2 Let $\gamma \leq \underline{q} /(2 \bar{p})$. Let $\zeta^{+}$be the positive root of the equation

$$
\frac{\bar{p}^{2}}{\underline{r}} \kappa_{C}^{2} \zeta^{2}+2 \bar{p} \kappa_{A} \zeta-(\underline{q}-2 \gamma \bar{p})=0
$$

Let $\rho=\min \left(\alpha, \zeta^{+}\right)$. Any trajectory of the system (5) that starts in the ball of center $\hat{x}(0)$ and constant radius $\rho / \sqrt{\bar{p}}$ with respect to the metric (6) remains in a ball of radius $\rho / \sqrt{\bar{p}}$ centered at the trajectory $\hat{x}(t)$ of the Extended Kalman Filter (3)-(4), and converges exponentially in the sense of the metric (6) with a time constant $1 / \gamma$ for the exponential decay. In particular, this implies in the Euclidean metric on vectors, that

$$
\|\hat{x}(t)-x(t)\| \leq \sqrt{\bar{p} / \underline{p}}\|\hat{x}(0)-x(0)\| \exp ^{-\gamma t}
$$

for all $t \geq 0$ as soon as initially $\|\hat{x}(0)-x(0)\| \leq \rho \sqrt{\underline{p}} / \sqrt{\bar{p}}$.

Proof The result directly follows from Theorem 1 as long as one can prove that $\rho \leq r(t)$ for all $t \geq 0$, where $r(t)$ is the radius of a ball around $\hat{x}(t)$ in which the matrix inequality (9) is verified. To begin, note that the ball of center $\hat{x}$ and radius $\zeta^{+}$is equivalently defined as the set

$$
\left\{z \in \mathbb{R}^{n}, 2 \bar{p} \kappa_{A}\|\hat{x}-z\|+\frac{\bar{p}^{2}}{\underline{r}} \kappa_{C}^{2}\|\hat{x}-z\|^{2} \leq \underline{q}-2 \gamma \bar{p}\right\}
$$

Let $\tilde{e}=z-\hat{x}$. As $\tilde{A}(z, t)=A(z, t)-A(\hat{x}, t)=\int_{0}^{1} \frac{\partial^{2} f}{\partial x^{2}}(\hat{x}+r \tilde{e}, t) \tilde{e} d r$ and $\tilde{C}(z, t)=C(z, t)-$ $C(\hat{x}, t)=\int_{0}^{1} \frac{\partial^{2} h}{\partial x^{2}}(\hat{x}+r \tilde{e}, t) \tilde{e} d r$, we have $\|\tilde{A}\| \leq \kappa_{A}\|\hat{x}-z\|$ and $\|\tilde{C}\| \leq \kappa_{C}\|\hat{x}-z\|$. The largest value of the symmetric matrix $\tilde{A} P+P \tilde{A}^{T}$ satisfies

$$
\begin{aligned}
\lambda_{\max }\left(\tilde{A} P+P \tilde{A}^{T}\right) & =\max _{\|w\|=1} w^{T}\left(\tilde{A} P+P \tilde{A}^{T}\right) w \\
& =2 \max _{\|w\|=1} \operatorname{Trace}\left(w^{T} \tilde{A} P w\right) \\
& \leq 2 \bar{p} \max _{\|w\|=1} w^{T} \tilde{A} w \\
& \leq 2 \bar{p} \max _{\|w\|=1,\|v\|=1}\left|w^{T} \tilde{A} v\right| \leq 2 \bar{p}\|\tilde{A}\| \leq 2 \bar{p} \kappa_{A}\|\hat{x}-z\|
\end{aligned}
$$

In the same way we have $\lambda_{\max }\left(P \tilde{C}^{T} R^{-1} \tilde{C} P\right) \leq \frac{\bar{p}^{2}}{\underline{r}} \kappa_{C}^{2}\|\hat{x}-z\|^{2}$. Thus, as long as $z$ belongs to the set (12), the inequality (9) is satisfied. 


\section{Links with previous work in the literature}

The Extended Kalman filter has been shown to converge locally exponentially under a set of conditions on the nonlinearities of the system, see e.g. [15, 5] in continuous-time and [16, 2, 1] in discrete-time. In these papers, the convergence analysis is based on the Lyapunov function

$$
V(x-\hat{x}, t)=(x-\hat{x})^{T} P^{-1}(t)(x-\hat{x})
$$

At fixed time $t, V(x-\hat{x}, t)$ is the geodesic distance between $x$ and $\hat{x}$ for the proposed metric

$$
\|\delta z\|_{P^{-1}}^{2}=\delta z^{T} P^{-1} \delta z
$$

Thus, it is no surprise that the convergence conditions derived in this article are very similar to those previously appearing in the literature. That said, the specificity of the contraction framework yields some differences that we shall detail in this section, which is organized as follows. In Subsection 4.1 we emphasize a difference of point of view between contraction analysis and Lyapunov based convergence analysis. In Subsection 4.2 we compare the convergence rate and basin of attraction of Section 3 with the results appearing previously in the literature. For fair comparison we chose the article 15] which deals with the continuous-time case. Finally, in Subsection 4.3, the modification of the EKF proposed in [15] is analyzed in the light of contraction theory, yielding a simple generalization of this work which is straightforward in our framework.

\subsection{A different approach}

In standard Lyapunov analysis of nonlinear deterministic observers, one generally seeks to prove the state error, i.e., the discrepancy between the estimated trajectory $\hat{x}$ and the true trajectory $x$, measured by some Lyapunov function, tends to zero. Often the observers can be only proved to be locally convergent, i.e., the initial guess $\hat{x}(0)$ must belong to some attraction basin containing $x(0)$. The contraction analysis of the present paper, based on the idea of virtual systems [17] and specifically virtual observers [8, builds upon a different approach. Indeed, the idea is to focus on a particular trajectory of the filter $\hat{x}(t), t \geq 0$, and then to study the evolution of two neighboring trajectories of the virtual system (5), and prove that the distance between them tends to shrink over the time. Under a set of conditions, we have proved that this property holds in a ball centered at the particular trajectory $\hat{x}(t), t \geq 0$.

The contraction results underlying Theorem 1 and its corollaries are natural properties to expect from any filter. Indeed, they prove that as a dynamical system the observer possesses stability properties, and this independently from the behavior of the true system, or modeling errors. To be more specific, it is proved two initially close enough trajectories of the filter equation will verify equation (10). This exponential forgetting of the initial condition is thus a very desirable property for filters, which indicates robustness with respect to bad guesses and perturbations. This property is valid whether the true trajectory $x(t)$ belongs to the contraction set or not.

Besides, contraction theory provides a concrete result on the robustness of the EKF against external perturbations. Suppose indeed that $x_{p}(t)$ is some trajectory of a perturbed virtual system $\frac{d}{d t} x_{p}=f\left(x_{p}, t\right)-K(t)\left(h\left(x_{p}, t\right)-y_{m}(t)\right)+b\left(x_{p}, t\right)$ where $b\left(x_{p}, t\right)$ represents a disturbance whose norm is supposed to be uniformly bounded by, say, $\|b\|_{\max }$. Then we have

(see [12, 3]): $\left\|x_{p}(t)-\hat{x}(t)\right\| \leq \sqrt{(\bar{p} / \underline{p})}\left(e^{-\frac{t}{\gamma}}\left\|x_{p}(0)-\hat{x}(0)\right\|+\gamma\|b\|_{\max }\right)$. It proves that any trajectory of the perturbed system converges exponentially to a ball of radius $\sqrt{(\bar{p} / \underline{p})} \gamma\|b\|_{\max }$ around the observer trajectory, allowing to evaluate the estimation error generated by the perturbation. The latter result completes the robustness properties of the EKF. 


\begin{tabular}{|l|c|r|r|}
\hline & Convergence rate & Attraction basin for $\kappa_{C}=0$ & Attraction basin for $\kappa_{A}=0$ \\
\hline Lyapunov & $\gamma=q p /\left(4 \bar{p}^{2}\right)$ & $\|x(0)-\hat{x}(0)\| \leq(\underline{p} / \bar{p})\left[\underline{q} /\left(4 \kappa_{A} \bar{p}\right)\right]$ & $\|x(0)-\hat{x}(0)\| \leq q \underline{r} /\left(4 \bar{c} \kappa_{C} \bar{p}^{2}\right)$ \\
\hline Contraction & $\gamma=q /(4 \bar{p})$ & $\|x(0)-\hat{x}(0)\| \leq(p / \bar{p})^{1 / 2}\left[\underline{q} /\left(4 \kappa_{A} \bar{p}\right)\right]$ & $\|x(0)-\hat{x}(0)\| \leq(q p \underline{r})^{1 / 2} /\left(\kappa_{C} \bar{p}^{3 / 2} \sqrt{2}\right)$ \\
\hline
\end{tabular}

Table 1: Comparison of convergence rate and attraction basin.

\subsection{Comparison of convergence results}

First of all, to the authors' knowledge Theorem 1 and its Corollary 1 have never appeared in the literature. The common approach is to study the evolution of the Lyapunov function (13) over time. While similar results to Theorem 1 and its corollary may possibly be worked out from this approach as well, they appear quite naturally in a contraction framework.

Consider now the results of Section 3. As already mentioned, the standard Lyapunov function (13) represents the geodesic distance between $\hat{x}$ and $x$ in the sense of the metric proposed in this paper. This is why the bounds derived in Section 2 are very similar to those previously obtained in the literature. However, they are different, and this is mainly due to the use of equation (7) in the result (8). Again, the analog of this transformation in the Lyapunov framework is not easily seen, whereas it appears naturally in the contraction framework.

Assume for simplicity that $\alpha=+\infty$. Table 1 compares the convergence rate and attraction basin obtained in $[15$ and the ones obtained in the present paper in the two limiting cases $\kappa_{A}=0$ and $\kappa_{C}=0$. The results all correspond to the error equation (11) in the Euclidean metric. The rates and bounds in the Lyapunov approach of [15] have been obtained letting $\alpha=0$ (this parameter has a different meaning in this article) and using the definition of $\kappa$ derived therein.

We see that the results are quite similar, yet different. First of all, we immediatly see that both the convergence rate and the size of the guaranteed attraction basin are larger in our approach in the limiting case $\kappa_{C}=0$, improving the formerly obtained bounds of [15]. Indeed $\gamma$ given in Table 1 is greater in the contraction case by a factor $\bar{p} / \underline{p} \geq 1$ (yielding faster convergence), and the size of the attraction basin by a factor $(\bar{p} / \underline{p})^{1 / 2} \geq 1$. The difference is much more remarkable in the limit case $\kappa_{A}=0$, in which case the size of the attraction basin depends heavily on the problem parameters. In particular, a noticeable difference is that the size of the attraction basin in [15] depends on $\bar{c}$, an upper bound on $\{\|C(t)\|, t \geq 0\}$. As a result, a large linearized observation matrix $C(t)$ can diminish the guaranteed size of the attraction basin. On the other hand, the bounds obtained in the present paper do not rely on an upper bound $\bar{c}$, and therefore they may yield stronger guarantees in some cases.

\subsection{A contraction-based interpretation of the observer of [15]}

In [15, the authors propose to modify slightly the EKF by adding a new term $2 \beta P$ in the Riccati equation (4), with $\beta \geq 0$, leading to a modified Riccati equation:

$$
\frac{d}{d t} P=A(\hat{x}, t) P(t)+P A(\hat{x}, t)^{T}+Q-P C(\hat{x}, t)^{T} R^{-1} C(\hat{x}, t) P+2 \beta P
$$

They prove the addition of such a term yields a faster convergence rate, as long as Assumption 1 is preserved. Note that, such a term tends to increase the eigenvalues of $P$ and thus to destabilize the Riccati equation. The fact that Assumption 1 remains then valid is thus non trivial and must be checked. Then, as long as Assumption 1 is proved to hold, this term ensures a faster convergence rate. This latter fact is easily understood in the contraction 
framework. Indeed, with this additional term, inequality (9) becomes:

$$
P \tilde{A}^{T}+\tilde{A} P+P \tilde{C}^{T} R^{-1} \tilde{C} P \leq Q-2(\gamma+\beta) P+P C^{T} R^{-1} C P
$$

The benefits of this term are now obvious as it transforms the convergence rate $\gamma$ into $\gamma+\beta$. In fact, contraction theory even offers a direct generalization of the work of 15. Indeed consider the following modified EKF observer

$$
\begin{aligned}
& \frac{d}{d t} \hat{x}=f(\hat{x}, t)-P C(\hat{x}, t)^{T} R^{-1}\left(h(\hat{x}, t)-y_{m}(t)\right) \\
& \frac{d}{d t} P=A(\hat{x}, t) P(t)+P(t) A(\hat{x}, t)^{T}+Q-P(t) C(\hat{x}, t)^{T} R^{-1} C(\hat{x}, t) P(t)+2 N
\end{aligned}
$$

where $N$ is any positive definite matrix. It follows directly from equation (9) that as long as Assumption 1 still holds, the guaranteed convergence rate of this observer is increased by adding $\underline{n} / \bar{p}$, where $\underline{n}$ denotes the smallest eigenvalue of $N$.

\section{Acknowledgements}

The authors would like to thank Laurent Praly for interesting feedback on the paper.

\section{References}

[1] J.S. Baras, A. Bensoussan, and M.R. James. Dynamic observers as asymptotic limits of recursive filters: special cases. SIAM J. Appl. Math., 48:1147-1158, 1988.

[2] M. Boutayeb, H. Rafaralahy, and M. Darouach. Convergence analysis of the extended Kalman filter used as an observer for nonlinear deterministic discrete-time systems. IEEE transactions on automatic control, 42, 1997.

[3] D. Del Vecchio and J.J. Slotine. A contraction theory approach to singularly perturbed systems. IEEE Trans. On Automatic Control. 2012. To appear.

[4] B.P. Demidovich. Dissipativity of a nonlinear system of differential equations. Vestnik Moscow State University, Ser. Mat. Mekh., 1961.

[5] J. Deyst and C. Price. Conditions for asymptotic stability of the discrete minimumvariance linear estimator. IEEE Trans. on Automatic Control, 13:702-705, 1968.

[6] F. Deza, J-P. Gauthier and E. Busvelle. High-gain estimation for nonlinear systems. Systems and Control Letters, 18:295-299, 1992.

[7] P. Hartman. On stability in the large for systems of ordinary differential equations. Canadian Journal of Mathematics, 13:480-492, 1961.

[8] J. Jouffroy and J.J.E. Slotine. Methodological remarks on contraction theory. In 43rd IEEE Conference on Decision and Control, 2004.

[9] R. Kalman and R. Bucy. New results in linear filtering and prediction theory. Basic Eng., Trans. ASME, Ser. D,, 83(3):95-108, 1961.

[10] A. Krener. Mathematical Systems Theory and Optimization, chapter The Convergence of the Extended Kalman Filter, pages 173-182. Springer Verlag, 2002. 
[11] D. C. Lewis. Metric properties of differential equations. Amer. J. Math., 71:294-312, 1949.

[12] W. Lohmiller and J.J.E. Slotine. On contraction analysis for nonlinear systems. Automatica, 34(6):683-696, 1998.

[13] W. Lohmiller and J.J.E. Slotine. Contraction Analysis of Nonlinear Distributed Systems. International Journal of Control, 78(9), 2005.

[14] D. Luenberger. An introduction to observers. IEEE Trans. on Automatic Control, 16(6):596-602, 1971.

[15] F. Sonnemann Reif, K. and R. Unbehauen. An EKF-based nonlinear observer with a prescribed degree of stability. Automatica, 34:1119-1123, 1998.

[16] Y.K. Song and J.W. Grizzle. The extended Kalman filter as a local asymptotic observer. Estimation and Control, 5:59-78, 1995.

[17] W. Wang and J.J.E. Slotine. On partial contraction analysis for coupled nonlinear oscillators. Biological Cybernetics, 92(1), 2005. 